CAHIERS DE

NARRATOLOGIE

\section{Cahiers de Narratologie}

Analyse et théorie narratives

$31 \mid 2016$

Sérialité narrative. Enjeux esthétiques et économiques

\title{
La Voix de Proust
}

Des influences de À la recherche du temps perdu sur la construction des notions de narration et de voix narrative chez Gérard Genette

\section{Olivier Stucky}

\section{(2) OpenEdition}

\section{Journals}

Electronic version

URL: http://journals.openedition.org/narratologie/7613

DOI: $10.4000 /$ narratologie. 7613

ISSN: $1765-307 X$

Publisher

LIRCES

\section{Electronic reference}

Olivier Stucky, "La Voix de Proust », Cahiers de Narratologie [Online], 31 | 2016, Online since 22

December 2016, connection on 01 May 2019. URL : http://journals.openedition.org/narratologie/7613 ; DOI : 10.4000/narratologie.7613

This text was automatically generated on 1 May 2019.

Article L.111-1 du Code de la propriété intellectuelle. 


\section{La Voix de Proust}

Des influences de À la recherche du temps perdu sur la construction des notions de narration et de voix narrative chez Gérard Genette

\section{Olivier Stucky}

Je ne sais pas si je vous ai dit que ce livre était un
roman. Du moins, c'est encore du roman que cela
s'écarte le moins. Il y a un monsieur qui raconte et
qui dit : «Je »

Lettre à René Blum du 23 février 1913, Marcel

Proust $^{1}$

\section{Introduction}

1 Dans Nouveau discours du récit ${ }^{2}$, Gérard Genette justifie son choix, vieux de plus de dix ans, d'articuler « Discours du récit ${ }^{3}$ » autour de À la recherche du temps perdu, en évoquant les rudes conditions météorologiques d'un hiver étasunien et d'une bibliothèque chichement fournie des seuls volumes Pléiade du roman de Marcel Proust ${ }^{4}$. L'anecdote est, certes, amusante et engagerait presque les destinataires de ce nouvel ouvrage - dont l'un des objectifs est de répondre aux différentes critiques reçues à l'adresse de DR - à se raviser face aux difficultés de rédaction rencontrées par un narratologue enseveli sous la neige. Pourtant, cette tentative de captatio benevolentiae ne tient pas dès lors que l'on considère le fait que l'emploi de la Recherche dans DR revêt des enjeux plus complexes que les simples hasards d'une bibliothèque mal garnie. Car, peu importent en fait les conditions biographiques qui auraient poussé Genette à sélectionner un texte plutôt qu'un autre. Le fait est que le DR a exercé et exerce encore une influence indéniable dans le domaine de la narratologie. Or, l'influence du corpus proustien sur cet article est fondamentale à sa compréhension et a, à notre sens, grandement participé à l'obsession communicationnelle de la narratologie $e^{5}$. 
2 D'un point de vue méthodologique, la posture qu'adopte Genette par rapport au récit proustien implique une intrication totale de l'objet dans la construction de son discours. Ainsi, la production narratologique à l'œuvre dans DR se voit complètement influencée par la Recherche et s'articule autour de complexités qui lui sont spécifiques, notamment celles liées à l'identification d'un narrateur à la première personne. Tout cela concourt à la construction d'un outillage théorique largement influencé tant par la narration proustienne que par le contexte intellectuel de l'époque ainsi que la méthode expérimentale à laquelle s'astreint Genette dans son article. Outillage dont la part consacrée aux problèmes de la "voix » feront l'objet de vives critiques auxquelles le Nouveau discours $d u$ récit vise à fournir une réponse. Toutefois, l'arbre des excuses préliminaires d'un hasard bibliographique auquel nous faisions référence ne fait que cacher la forêt d'une vaste problématique pour la narratologie.

3 En effet, il semble que les critiques les plus importantes à l'égard de la théorie genettienne se soient concentrées autour de cette question précise de la narration ${ }^{6}$, notamment au sein du pan non-communicationnaliste $e^{7}$ de la discipline. Les commentaires initiés par Ann Banfield dans Phrases sans paroles ${ }^{8}$ sont repris par Sylvie Patron dans Le Narrateur' où l'attaque à l'égard de Genette est frontale ${ }^{10}$. Cependant, si la discorde prend souvent appui sur le fait que la théorie genettienne présuppose l'identification d'un narrateur anthropomorphe, de toute évidence, une réflexion quant à la construction de sa notion de narration par le recours à une étude détaillée de la partie consacrée à la voix manque à l'appel. L'hypothèse que nous défendrons consiste à postuler que l'engendrement des paramètres constituant la voix narrative chez Genette est au moins en grande partie conditionné par son rapport avec le récit proustien. Partant du principe que Gérard Genette construit son article autour d'une perspective théorico-critique de la Recherche, il s'agira ici d'évaluer à quels niveaux le choix et la perspective de son corpus influencent l'élaboration théorique des différents aspects de cette notion.

4 Ainsi, nous articulerons notre propos en trois temps. Premièrement, il s'agira de ressaisir les objectifs générateurs de DR. La méthodologie à laquelle Genette propose de se soumettre dans son article sera ainsi étudiée tant dans son inscription au sein du contexte intellectuel de la fin des années soixante, que de la manière dont il en fait l'exposition dans l'avant-propos de son article. Deuxièmement, nous observerons comment cette méthode se voit mise en œuvre dans DR et montrerons qu'en dehors même du développement consacré à la notion de narration, le corpus proustien mène dans cette direction et complexifie ainsi l'élaboration théorique. Enfin, troisièmement, nous nous pencherons plus précisément sur la partie de l'article consacrée à la voix, afin de voir comment les axes problématiques établis dans les parties précédentes interviennent dans la construction théorique de la narration selon Gérard Genette.

\section{D’un mélange entre critique et théorie}

Comme nous l'avons déjà évoqué, au sein même de son projet méthodologique, DR pose des difficultés d'approche en se situant explicitement dans une double démarche de critique et de théorie, dont Genette détaille les implications respectives. De son point de vue, la perspective théorique implique de «[mettre] franchement, comme d'autres l'ont fait ailleurs, l'objet spécifique au service de la visée générale, et l'analyse critique au service de la théorie » en prenant en compte le risque que «la Recherche ne [soit] plus ici qu'un prétexte, réservoir d'exemples et lieu d'illustration pour une poétique narrative où 
ses traits spécifiques se perdraient dans la transcendance des "lois du genre" ». Au contraire, l'approche critique, " en [lui] subordonnant au contraire la poétique » a pour conséquence de faire « des concepts, des classifications et des procédures proposés [dans l'article] autant d'instruments ad hoc exclusivement destinés à permettre une description plus exacte ou plus précise du récit proustien dans sa singularité, le détour "théorique" étant à chaque fois imposé par les nécessités d'une mise au point méthodologique » (DR : 68). Exposition de deux postures face auxquelles Genette refuse de trancher: «avou[ant] [s]a répugnance, [s]on incapacité, entre ces deux systèmes de défense apparemment incompatibles » (ibid.). L'exposition de ces deux approches est à recontextualiser. La question de cette dissociation est, en effet, d'actualité dans le milieu structuraliste de la critique littéraire à la fin des années $1960^{11}$, et elle se voit donc problématisée chez Genette dans une tentative d'enchevêtrement en vue de produire son essai. La frontière entre les deux approches est ainsi très clairement exposée par Roland Barthes dans Critique et véritét ${ }^{12}$ où les deux démarches servent des visées radicalement opposées. Aussi, il s'agira, pour nous, d'identifier les enjeux théoriques d'un tel assemblage, entre la perspective d'une narratologie générale et l'étude spécifique du récit proustien.

Précisons ici que nous adoptons un point de vue théorique sur son travail et qu'en cela, nous privilégions cette perspective au détriment de son approche critique.

6 Ainsi, il est donc nécessaire de ressaisir, pour mieux la comprendre, la posture intellectuelle adoptée par Genette qu'induit la publication de cet article. La discussion qu'il engage par le biais de cette démarche critique et théorique entre en dialogue avec une réflexion qui se rattache à l'actualité de la deuxième moitié des années 1960, dans le mouvement de fondation d'une "science de la littérature » contre l'approche historique et biographiste prônée par l'académie ${ }^{13}$. En effet, dans le projet barthésien, la critique littéraire se dissocie radicalement de la théorie afin d'attribuer à chacune de ces deux disciplines un champ d'action et une méthodologie qui lui sont propres. Le cahier des charges de cette nouvelle perspective est ainsi défini :

Ce ne pourra être une science des contenus (sur lesquels seule la science historique la plus stricte peut avoir prise), mais une science des conditions du contenu, c'est-àdire des formes : ce qui l'intéressera, ce seront les variations de sens engendrées, et, si l'on peut dire, engendrables, par les œuvres: elle n'interprétera pas les symboles mais seulement leur polyvalence; en un mot, son objet ne sera plus les sens pleins de l'œuvre, mais au contraire le sens vide qui les supporte tous ${ }^{14}$.

7 On reconnaît là les deux postures décrites par Genette. Cette volonté de distribution des tâches n'est de loin pas isolée dans le champ des études littéraires et se retrouve explicitée également chez Tzvetan Todorov:

La poétique vient rompre la symétrie ainsi établie entre interprétation et science dans le champ des études littéraires.

Par opposition à l'interprétation d'œuvres particulières, elle ne cherche pas à nommer le sens mais vise la connaissance des lois générales qui président à la naissance de chaque œuvre. Mais par opposition à ces sciences que sont la psychologie, la sociologie, etc., elle cherche ces lois à l'intérieur de la littérature même. La poétique est donc une approche de la littérature à la fois «abstraite » et « interne ${ }^{15}$ ».

Ici, la discipline poétique se situe à un degré plus éloigné des sciences que chez Barthes avec lesquelles il marque une distance. Il n'empêche que la démarche annoncée par Genette dans son avant-propos vient clairement rediscuter les enjeux d'une telle question, qu'il traite, par ailleurs, à travers d'autres articles compilés au sein de Figures III 
16. Par conséquent, il paraît inévitable d'envisager DR à la lumière de cette donnée qui situe l'article le plus important du recueil dans une démarche quasi expérimentale. Faire communiquer, comme il l'entend, les approches critiques et théoriques pour produire un essai narratologique, dont les présupposés se revendiquent justement de l'instauration d'une « science ${ }^{17}$ ", relève d'une tentative presque provocatrice qu'il s'agit de prendre en compte.

Quant à l'inscription de la Recherche au sein de ce projet, celle-ci est annoncée comme l'aspect fondateur du $\operatorname{propos}^{18}$. L'indécision entre approche critique et théorique est d'ailleurs justifiée par la complexité du roman :

Il me parait impossible de traiter la Recherche du temps perdu comme un simple exemple de ce qui serait le récit en général, ou le récit romanesque, ou le récit de forme autobiographique, ou Dieu sait quelle autre classe, espèce ou variété : la spécificité de la narration proustienne prise dans son ensemble est irréductible, et toute extrapolation serait ici une faute de méthode; la Recherche n'illustre qu'ellemême. (DR : 67)

La Recherche, est présentée a priori comme un objet singulier qui ne pourrait constituer le point de départ d'une théorie. Par ailleurs, rappelons avec Dominique Maingueneau l'importance que revêt l'œuvre de Proust pour toute cette génération de critiques. Dans son ouvrage Contre Saint Proust ${ }^{19}$, Maingueneau fait état de l'influence qu'a exercé la littérature proustienne sur la critique littéraire française. Ainsi, le mouvement engagé dès les années 1950, qui visait un renouvellement de la réflexion sur la littérature que l'on a appelé nouvelle critique, s'est-il saisi de l'œuvre de Proust pour porter ses idées :

Le nom de Proust se retrouve à tous les points stratégiques. Il a radicalisé la mythologie romantique de l'artiste, construisant méticuleusement dans sa vie et dans son œuvre cette mort de l'auteur que proclame l'épistémologie structuraliste. Ennemi de l'histoire littéraire, fondateur de la critique thématique et défenseur présumé des «structures ", il vient attester le bien-fondé de son rôle prédominant par le caractère génial de son œuvre romanesque, qui constitue précisément un objet d'analyse privilégié pour la Nouvelle critique ${ }^{20}$.

11 Le choix de la Recherche dans le travail de Genette n'est donc pas tout à fait un hasard. Proust innerve la critique littéraire des années 1960 et ce depuis la parution inédite chez Gallimard de Jean Santeuil en 1952 et du Contre Sainte Beuve en 1954, la même année où paraissent, pour la première fois à la Pléiade, les volumes de la Recherche - ceux-là même qui accompagneront manifestement Gérard Genette dans l'hiver étasunien de 1969. Aussi, la prise en compte dans $\mathrm{DR}^{21}$ non seulement du grand roman de Proust, mais aussi de l'ensemble de ses œuvres littéraires et de ses essais, constitue un indice de l'influence de la nouvelle critique, qui avait été fortement influencée par les idées professées dans Contre Sainte Beuve ${ }^{22}$.

Aussi, tant par le choix du corpus que par sa méthode, Genette vient se situer autant dans la continuité qu'en rupture avec une certaine posture théorique dans le champ des études littéraires. En se positionnant autant du côté théorique que de celui de la critique, Genette propose une approche en tension entre deux objectifs distincts. D'une part, DR est à envisager comme un essai narratologique ; d'autre part, il se définit comme un texte critique entendant démontrer la singularité du roman proustien au sein de la littérature. Par ailleurs, le choix de Proust comme point de départ de son essai met en évidence certains présupposés théoriques qui influenceront son approche. Les répercussions de la littérature proustienne sur la nouvelle critique, notamment le décret de la «mort de l'auteur ${ }^{23}$ » pointé du doigt par Maingueneau, ont une incidence sur les partis pris 
narratologiques qui parcourent DR. Cette influence est particulièrement manifeste dans la construction de la notion de narration, largement motivée par la présupposition que l'auteur est absent de son texte ${ }^{24}$. La tension entre les aspects théorique et critique de DR est constitutive de la démarche dans son ensemble, et toute étude approfondie de cet essai devrait en tenir compte. Car à ne considérer DR que comme un texte théorique, et, par conséquent, en tablant sur la valeur générale de toutes les notions qui y sont mises en place, on oublie que celles-ci répondent aussi à un objectif critique, qui visait à valoriser la singularité du récit proustien. Autrement dit, critique et théorie constituent les deux faces d'une même pièce. Aussi, dans le cas qui nous intéresse, ignorer la critique proustienne dans l'appréhension de la notion de narration, c'est oublier que sa construction vise la description d'un récit problématisant la communication du récit littéraire, ainsi que nous le mettrons en évidence. La prise de conscience de tels enjeux pour une étude de la constitution d'un discours partiellement théorique devient dès lors essentielle pour réfléchir à la mise en place des outils d'une « technologie » (DR: 269) du récit dont l'influence n'est plus à démontrer.

\section{Mise en œuvre du projet}

13 Ainsi que la prise en compte du contexte historique l'a montré, il importe de comprendre comment l'essai, en tant que discours théorique, articule son rapport au projet critique. En ceci, tant la structure générale de l'essai qu'une étude spécifique du développement d'une de ses parties nous renseignent quant aux implications d'un tel mélange méthodologique. À cet égard, et en premier lieu, l'observation de la structure de DR permet d'identifier une organisation textuelle visant à pondérer presque systématiquement les propositions théoriques par leur confrontation avec les spécificités du récit proustien. Ainsi, la section consacrée à l'ordre (DR: 77-121) ouvre sur «Vers l'achronie » (DR: 115-121), faisant état de la complexité des anachronies dans la Recherche. De même, l'analyse de la fréquence (DR : 145-182) se clôt par « Le jeu avec le Temps » (DR : 178-182) (la référence proustienne est évidente) ; enfin, l'étude du mode (DR : 183-224) mène à une proposition quant à la "Polymodalité » (DR : 214-224) où Genette discute les enjeux de la focalisation dans le régime narratif proustien. Nous ne mentionnons pas ici les études de la voix et de la durée, qui feront chacune l'objet d'un développement plus détaillé. L'ensemble de DR se construit ainsi autour d'un mouvement d'exposition théorique suivi, quasi systématiquement, d'une évaluation critique de l'œuvre de Proust, dont la prétendue singularité vient constamment bouleverser les catégories générales précédemment introduites.

Le cas de la réflexion au sujet des mouvements de durée face au récit proustien constitue un exemple significatif de la posture méthodologique adoptée par Genette dans DR et ce pour deux raisons. D'une part, parce qu'elle synthétise de manière manifeste le projet de mise en échec d'une théorie générale pour l'analyse du récit proustien. D'autre part, parce que, bien que traitant de la représentation du temps, la démonstration de Genette tend à situer l'essentiel des spécificités de la Recherche dans son rapport à l'acte de narration. Dans cette partie de l'article, la démonstration de Genette vise explicitement la conclusion que la narration proustienne ne se déroule qu'à la vitesse de la scène et génère, par son exemple seul, la typologie des opérations de fréquence (DR : 145-182), dont Genette souligne le caractère novateur ${ }^{25}$. La typologie des phénomènes de durée, articulée autour du « sommaire ", de la " pause », de l'« ellipse » et de la « scène », se construit comme la 
démonstration de l'inefficacité de chacune de ces catégories pour décrire la représentation du temps dans la Recherche. La disqualification systématique de la grille « traditionnelle » (DR : 129) d'analyse des variations de durée permet à Genette d'affirmer que l'ensemble de cette typologie fondée sur la tradition romanesque perd de sa pertinence pour l'analyse du récit proustien. Aussi, affirmer que «la totalité du texte narratif proustien peut se définir comme scène» (DR : 141) lui permet d'une part de mettre l'accent sur le récit comme acte de narration (qui ne s'interrompt et ne s'efface donc jamais) mais surtout de montrer que les modulations temporelles ne passent pas par le système romanesque conventionnel et nécessite son propre outillage d'analyse. Aussi conclut-il le chapitre en affirmant :

On voit donc que le récit proustien ne laisse intact aucun des mouvements narratifs traditionnels, et que l'ensemble du système rythmique de la narration romanesque s'en trouve profondément altéré. Mais il nous reste à connaître une dernière modification, la plus décisive sans doute, dont l'émergence et la généralisation vont donner à la temporalité narrative de la Recherche une cadence toute nouvelle - un rythme proprement inouï. (DR : 144)

Notons par ailleurs que la révocation complète de l'outillage théorique est motivée ici par une valorisation explicite de la Recherche qui semble opposer son génie à la pertinence des catégories de durée. Le caractère évaluatif de la proposition clôturant le paragraphe indique très clairement l'objectif de singularisation du roman de Proust, son « rythme proprement inouï » vient situer l'œuvre en rupture complète avec la tradition et la place au cœur du problème de l'analyse de la temporalité narrative.

Dans le cas particulier de la "pause » (DR: 133-138), sa disqualification passe par une réinterprétation générale du récit proustien. Bien que les descriptions, qui sont le lieu habituel de ce type de régime, soient bel et bien présentes chez Proust, pour Genette: «Une conclusion s'impose donc: c'est que la description [...], se résorbe en narration, et que le second type canonique de mouvement - celui de la pause descriptive - n'y existe pas, pour une évidente raison que la description y est tout sauf une pause du récit. » (DR : 138) Prendre le parti d'exclure la pause descriptive du récit proustien constitue un geste théorique pouvant paraître étonnant concernant un récit qui présente, de prime abord, de longues phases de description. Cependant, étant donné que DR se construit dans un entrelacement de critique et de théorie, cette conclusion "s'impose " puisque l'objectif systématique consiste à construire des catégories dont le roman proustien se fait le contre-exemple. Par ailleurs, le développement qui mène à cette conclusion présente un autre aspect de la perspective genettienne dans laquelle l'éviction de la pause témoigne d'une modalité narrative à part entière. En effet, pour Genette :

[M]ême quand l'objet décrit n'a été rencontré qu'une fois (comme les arbres de Hudimesnil), ou quand la description ne concerne qu'une seule de ses apparitions (généralement la première, comme pour l'église de Balbec, le jet d'eau Guermantes, la mer à la Raspelière), cette description ne détermine jamais une pause du récit, une suspension de l'histoire ou, selon le terme traditionnel, de l'« action »: en effet jamais le récit proustien ne s'arrête sur un objet ou un spectacle sans que cette station corresponde à un arrêt contemplatif du héros lui-même [...], et donc jamais le morceau descriptif ne s'évade de la temporalité de l'histoire. (DR : 134)

Le problème que pose la description proustienne met en évidence celui de la représentation d'une narration. Pour Genette, la pause descriptive chez Proust ne peut être une pause du récit parce que le narrateur ne rompt jamais tout à fait son discours. Dès lors, la distinction entre deux «modes » de narration est, dans une telle perspective, difficilement identifiable. La singularisation de l'objet que commande le projet critique 
dévoile la problématique fondamentale de la narration proustienne qui brouille la construction de l'outillage théorique. En cela, la conclusion apportée à l'étude de l'ellipse (DR : 139-141) est d'autant plus significative en ceci que, bien que Genette identifie des occurrences du phénomène dans la Recherche, l'intention de disqualification des catégories «standards " pour l'analyse du récit proustien le force à se détourner du problème. En effet, pour lui : «Si l'on considère le fait que les ellipses, quels que soient leur nombre et leur puissance d'élision, représentent une part du texte pratiquement nulle, il faut bien en venir à cette conclusion que la totalité du texte narratif proustien peut se définir comme scène [...]» (DR : 142) Certes, si, comme il le formule, l'ellipse correspond à une absence de narration, on pourrait ne pas la considérer comme prenant part de sa durée. Il n'empêche que ces sauts temporels participent d'une rythmique narrative en provocant des ruptures. Considérer que ces vides n'entrent pas en ligne de compte pour tirer des conclusions quant aux mouvements de durée revient à ne se concentrer que sur la narration en tant que production manifeste et à se refuser une réflexion sur le rôle de ces «absences » dans le récit.

Ainsi, l'étude de cette partie de DR montre que si la critique proustienne court-circuite quelque peu la théorie du récit, c'est notamment par le truchement des problématiques liées à la narration dans la Recherche. L'organisation du propos de Genette, articulé systématiquement autour de deux perspectives de réflexion, laisse transparaître le problème fondamental du choix d'un tel objet pour la construction d'une théorie générale du récit. Aussi, la partie consacrée à la voix, dont l'objectif est d'interroger les paramètres déterminant l'instance narrative, fera l'objet d'une analyse plus détaillée, afin de voir comment cette notion y est abordée à la lumière de la problématique que nous avons déjà mise en évidence.

\section{Une construction complexe : de l'influence de Proust sur la génération théorique}

Si le problème de la narration parcourt DR, l'étude du chapitre spécifiquement consacré à cette problématique permettra de mieux comprendre, d'une part, comment celle-ci est envisagée et, d'autre part, comment le projet théorique de Genette se voit complexifié par sa perspective critique.

Comme le remarque à juste titre Mieke Bal, au sein du triptyque notionnel défini dans l'introduction de DR (71-72), la notion de narration ne se conforme pas à l'unité homogène de la série : "[l]a narration [remarque-t-elle] concerne le procès d'énonciation, tandis que les deux autres concernent le produit d'une activité: l'histoire, qui est le produit de l'invention, et le récit, qui est pour Genette le produit de la disposition et de la narration ${ }^{26}$ . » Chez Genette, la narration se définit en effet comme « l'acte narratif producteur et, par extension, l'ensemble de la situation réelle ou fictive dans laquelle il prend place » (DR : 72). Ici comme ailleurs, les enjeux de la critique proustienne viennent complexifier le modèle théorique. En effet, l'adjonction de la notion de narration au duo récit/histoire, motive l'intervention de l'étude de la voix face à la division proposées par Todorov en « temps ", « mode » et " aspect ${ }^{27}$ ». Si Genette complexifie l'étude du « temps " par le biais des trois types de modifications temporelles que sont l'ordre, la durée et la fréquence et qu'il rabat l'aspect dans l'étude du mode, sa réflexion sur la voix, en tant que « rapport entre narration et récit» (DR : 76) est essentiellement motivée par le corpus proustien. En 
effet, l'étude de ces rapports entre récit et narration semble impliquer que cette dernière, en tant qu'acte producteur, puisse toujours être identifiée dans le texte. Autrement dit, que les énoncés qui le composent renvoient explicitement ou implicitement à leur situation d'énonciation, comme c'est le cas chez Proust :

Quant à la narration productrice de ce récit, l'acte de Marcel racontant sa vie passée, on se gardera dès maintenant de le confondre avec l'acte de Proust écrivant la Recherche du temps perdu [...] C'est donc le récit, et lui seul, qui nous informe ici, d'une part sur les événements qu'il relate, et d'autre part sur l'activité qui est censée le mettre au jour : autrement dit, notre connaissance des uns et de l'autre ne peut être qu'indirecte, inévitablement médiatisée par le discours du récit, en tant que les uns sont l'objet même de ce discours et que l'autre y laisse des traces, marques ou indices repérables et interprétables, tels que la présence d'un pronom personnel à la première personne qui dénote l'identité du personnage et du narrateur, ou celle d'un verbe au passé qui dénote l'antériorité de l'action racontée sur l'action narrative, sans préjudice d'indications plus directes et plus explicites. (DR : 73-74)

21 Ici encore, le modèle de la Recherche, qui fait de l'acte de narration littéraire l'un des enjeux spécifique du récit, façonne la théorie de telle manière qu'elle s'attarde sur des spécificités et non des généralités. Genette relève ici des exemples d'embrayages manifestes pour identifier le phénomène afin de justifier son approche. Cependant, il passe sous silence les nombreux cas où aucun marquage énonciatif n'est identifiable et ne motive donc pas un questionnement quant à l'identité du narrateur. Nous n'entendons pas dire ici que les notions développées par Genette au sujet de la voix n'ont pas de pertinence d'analyse pour bon nombre de récits. Toutefois nous insistons sur la perspective qui vise à axer la problématique sur des enjeux communicationnels, impliqués par l'analyse d'un récit énoncé par un "Je » dont le référent se dévoile progressivement, et qui mènent à postuler que «toute narration est, par définition, virtuellement faite à la première personne " (DR : 252, nous soulignons). Le problème se situe donc bien dans la définition de la narration comme part constitutive du discours du récit en tant qu'elle impose de postuler ce que le récit ne dit pas forcément. Ainsi, on voit que la notion même de narration revêt un potentiel problématique qui entre en discussion avec le projet complexe de Genette. En ceci, l'ensemble de la partie consacrée à la voix concentre les éléments les plus révélateurs de l'influence qu'exerce la critique proustienne sur la génération théorique.

22 Précisons d'emblée qu'il n'y a pas chez Genette de distinction fonctionnelle entre la notion de narration et de voix. Cette dernière n'est en fait qu'une dénomination grammaticale métaphorique justifiée par une logique de raccord avec le temps et le mode (DR : 76).

23 Afin d'articuler clairement notre propos, il est nécessaire d'opérer un classement hiérarchique des sous-parties consacrées à l'étude de la voix. Cette partie de l'article s'organise autour de ce que nous identifions comme cinq aspects de la narration par le biais de dix sous-parties distinctes. Passée l'introduction ${ }^{28}$, Genette se penche premièrement sur ce qu'il appelle le temps de la narration ${ }^{29}$, traitant du moment de l'énonciation narrative par rapport aux événements de l'histoire. Deuxièmement, il propose une réflexion sur ce que nous synthétiserons comme les niveaux de narration ${ }^{30}$, qui ont pour but d'établir le niveau auquel se situe le narrateur par rapport à la diégèse. Troisièmement, il s'interroge sur les relations diégétiques ${ }^{31}$ qui s'opèrent à travers les liens qu'entretient le narrateur avec la diégèse. Ces trois premiers aspects sont centraux pour 
notre propos et nous laisserons de côté ceux traitant des « fonctions du narrateur ${ }^{32}$ » et du « narrataire ${ }^{33}$ ", qui nous semblent périphériques par rapport à notre problématique ${ }^{34}$.

Le cas du temps de la narration est sans doute le plus révélateur du projet genettien en ceci qu'il s'organise très nettement en deux temps de réflexion distincts. Ainsi, son paragraphe introductif (DR: 228) est-il lui-même organisé entre une première appréhension générale, suivie d'une réflexion sur le cas proustien en particulier. Le paragraphe présente ainsi les aspects principaux de la réflexion qui entend répondre à des problématiques de narration littéraire auxquelles est confrontée ensuite la Recherche. Le cahier des charges de Genette est ici explicite : construire une théorie générale pour servir sa réflexion sur le récit proustien, ainsi qu'il l'expose en conclusion de son paragraphe introductif :

[I]l nous importe beaucoup de savoir, par exemple, combien de temps s'écoule entre la première scène de la Recherche (le "drame du coucher ") et le moment où elle est évoquée en ces termes: « Il y a bien des années de cela. La muraille de l'escalier où je vis monter sa bougie n'existe plus depuis longtemps, etc. »; car cette distance temporelle, et ce qui la remplit, et ce qui l'anime, sont ici un élément capital de la signification du récit. (DR : 228)

Ici, comme partout, la finalité critique prime sur le développement théorique. Qu'il importe beaucoup de connaître le temps qui s'écoule entre l'enfance du narrateur et le moment où il énonce son récit est une interrogation qui intéresse spécifiquement l'analyse proustienne. Alors que cette question ne se pose pas dans bon nombre de récits, elle est centrale chez Proust, parce que le roman la problématise au sein de son dispositif narratif, ainsi que le glose Genette à l'ouverture de sa réflexion sur la voix :

«Longtemps je me suis couché de bonne heure »: de toute évidence cet énoncé ne se laisse pas déchiffrer - comme, disons, "L'eau bout à cent degrés » ou « La somme des angles d'un triangle est égale à deux droits » - sans égard à celui qui l'énonce, et pour la situation dans laquelle il l'énonce; je n'est identifiable que par référence à lui, et le passé révolu de l'«action » racontée n'est tel que par rapport au moment où il la raconte. (DR : 225)

Partir de l'énoncé inaugural de la Recherche permet de cerner d'emblée les préoccupations fondamentalement communicationnelles de Genette, qui se reflèteront dans sa théorisation du récit. Mais on peut légitimement se demander si l'on se poserait la même question à la lecture des incipits de L'Education sentimentale de Flaubert ou de n'importe quel opus des Rougon-Macquart de Zola. Le cadre critique limite manifestement la perspective générale. La question du temps de la narration est certes un paramètre à décrire, mais son développement se voit complètement contrôlé par le projet critique. Ainsi, même du point de vue méthodologique, la hiérarchisation du développement des types de rapports temporels entre la narration et l'histoire sont contraints par la nécessité de garder l'exemple de Proust en ultime exposition. Les quatre types de relation identifiés par Genette (" antérieure », " simultanée », « intercalée » et " postérieure ») ne sont ainsi pas développés selon un ordre logique (soit de complexité, de rareté voire simplement chronologique) mais s'organisent dans le seul but de faire figurer la narration postérieure en bout de chaîne, afin d'assurer la jointure avec la partie consacrée à la Recherche (DR : 234-238). Et bien qu'ici l'exemple proustien ne vient pas ébranler le système canonique, reste que la démarche même de la démonstration prend pour principe que tout récit engendre une volonté d'identification d'un acte narratif attribué à une instance anthropomorphe. Genette trouve dans la Recherche de quoi instaurer en cas général ce que le roman a de particulier. Aussi, on remarquera que le rapport instauré à l'égard de son objet est ambivalent. Soit, comme dans le cas des catégories de durée, la 
Recherche sert à infirmer l'ensemble d'un modèle d'analyse, soit elle génère des outils théoriques par son seul exemple. Cependant, dans un cas comme dans l'autre, le roman de Proust est au centre du mouvement. Ainsi, la disqualification des types de durée sert à construire l'analyse des phénomènes de fréquence. À l'arrivée la démarche de Genette place toujours la Recherche au cœur du problème, tant pour invalider une typologie tirée de la tradition narrative littéraire (DR : 128-129) que pour en construire une nouvelle en généralisant les phénomènes repérés dans le texte. Dans le cas qui nous intéresse ici, Genette injecte dans des catégories générales des préoccupations toutes proustiennes. S'il est évident que les narrations intercalées ou simultanées mettent en scène un acte narratif, les cas de la narration antérieure et, surtout, postérieure, mettent en évidence un décalage entre les événements racontés et le récit qui ne font pas nécessairement l'objet d'un questionnement du même ordre que ce qu'annonce l'incipit de la Recherche. L'attention que porte Genette à la complexité du récit proustien le pousse à complexifier tout acte de narration pour en justifier la singularité. Ainsi, avant d'engager l'analyse de la temporalité de la narration chez Proust, il propose cette synthèse :

Contrairement à la narration simultanée ou intercalée, qui vit de sa durée et des relations entre cette durée et celle de l'histoire, la narration ultérieure vit de ce paradoxe, qu'elle possède à la fois une situation temporelle (par rapport à l'histoire passée) et une essence intemporelle, puisque sans durée propre. Comme la réminiscence proustienne, elle est extase, "durée d'un éclair», miraculeuse syncope, « minute affranchie de l'ordre du Temps ». (DR : 234)

Il ne fait pas de doute que, dans le cas de la Recherche, la question de la temporalité de la narration constitue un enjeu manifeste. Cependant, d'un point de vue théorique, cette remarque ne fait que complexifier un problème qui ne se pose qu'à un degré très anecdotique, comme dans l'exemple de Madame Bovary commenté par Genette (DR : 232-234). Plus encore, il est fréquent que le problème ne se pose pas du tout, à l'exemple de bon nombre de romans français de la seconde moitié du XIX ${ }^{\mathrm{e}}$ siècle dans lesquels le rapport temporel du narrateur avec l'histoire n'est pas problématisé.

$\mathrm{Si}$, ainsi que nous l'avons vu, Genette complexifie souvent son modèle théorique au profit de sa critique du roman de Proust, dans le cas du temps de la narration, le phénomène est encore plus visible et il est encore plus extrême dans les sous chapitres consacrés aux niveaux. Si la dichotomie qu'il pose entre narrateur extra- et intradiégétiques a fait l'objet de nombreuses réserves, dont il fait état dans Nouveau discours $d u$ récit ${ }^{35}$, ces commentaires semblent avoir essentiellement porté sur des problèmes de compréhension du modèle. Pourtant, encore une fois, l'aspect le plus problématique concerne l'orientation du développement théorique. En effet, s'il n'y a pas grand-chose à redire de sa dichotomie, l'objectif de son analyse peut porter à confusion. À cet égard, le développement consacré au pseudo-diégétique (DR : 246-251) interpelle par sa complexité. L'instauration de ce concept vient interférer dans la dichotomie intra-/extradiégétique en proposant une catégorie taillée sur mesure pour la Recherche. En classant les métalepses selon deux catégories - métadiégétique et pseudo-diégétique (DR: 243-246)-, Genette construit la seconde sans pour autant fournir un corpus solide d'exemples ${ }^{36}$. Force est donc de constater que le récit pseudo-diégétique est en fait, dans une certaine mesure, réservé d'avance à la description du récit proustien. Genette l'annonce d'ailleurs en guise de conclusion de la partie ouvrant celle consacrée au «triomphe du pseudo-diégétique » dans le passage de Jean Santeuil à la Recherche :

Partout ailleurs, en revanche, la pratique constante du récit dans la Recherche est ce que nous avons baptisé le pseudo-diégétique, c'est-à-dire un récit second en son 
principe, mais immédiatement ramené au niveau premier et pris en charge, quelle qu'en soit la source, par le héros-narrateur. La plupart des analepses relevées au premier chapitre procèdent soit de souvenirs remémorés par le héros, et donc une sorte de récit intérieur à la manière nervalienne, soit de relations qui lui ont été faites par des tiers. (DR : 248-249)

Proposition discutable en tant que l'on peut se demander à quel titre des récits assumés par un seul et unique narrateur au sein d'un flux narratif constant peuvent être catégorisés comme "seconds ", d'autant mieux que leur spécificité est d'être « ramené[s] au premier niveau » et assumés par le narrateur extra-diégétique... qui se trouve, au final, être une seule et même instance. Bien qu'il ne soit pas question ici de nier l'utilité de la notion de narration pseudo-diégétique, il parait nécessaire d'émettre quelque doute quant à sa rentabilité théorique en regard de la fonction critique qu'elle prend chez Genette pour défendre la singularité du récit proustien. Que l'on puisse catégoriser un récit de seconde main pris en charge par le narrateur extra-diégétique est certes productif, notamment dans le cas proustien d'Un Amour de Swann, dont l'économie narrative détonne d'avec le reste du roman et engage automatiquement un questionnement pour le lecteur. Ce qui est toutefois plus discutable c'est l'importance que Genette réserve à cette catégorie, qu'il semble considérer comme omniprésente chez Proust, dans l'objectif manifeste de singulariser la Recherche. À cet égard, les deux souscatégories qu'il attribue à cette notion (récits intérieurs et récits « de seconde main ») sont illustrées par des cas discutables. Dans le premier cas Genette propose l'exemple suivant :

Ressortissent au premier type, par exemple, les dernières pages des Jeunes Filles en fleurs qui évoquent les matinées ensoleillées de Balbec, mais à travers le souvenir qu'en a gardé le héros rentré à Paris : «Ce que je revis presque invariablement quand je pensai à Balbec, ce furent les moments où, chaque matin, pendant la belle saison... »; après quoi l'évocation oublie son prétexte mémoriel et se développe pour elle-même, en récit direct, jusqu'à la dernière ligne, en sorte que bien des lecteurs ne remarquent pas le détour spatio-temporel qui lui avait donné naissance, et croient à un simple "retour en arrière » isodiégétique, sans changement de niveau narratif. (DR : 249)

Difficile de voir dans cet exemple de À l'ombre des jeunes filles en fleurs une quelconque forme de récit second. Ici, il semble que Genette distorde quelque peu son modèle au profit de l'identification d'une spécificité proustienne. Il n'y a pourtant pas lieu de voir, à notre avis, une quelconque dissociation de deux actes narratifs individuels que le narrateur extra-diégétique ferait mine de prendre à sa charge, et ce dans la mesure où il n'y a pas eu d'acte narratif commis à proprement parler, mais plutôt une remémoration dont le narrateur fait état. Le problème auquel se confronte Genette est conséquence du brouillage énonciatif mis en scène par le texte proustien. Dans un passage comme celuilà, l'identification des niveaux de narration est en effet troublée par le dispositif mobilisé pour faire état de cet épisode de la vie du narrateur. De ce point de vue, il est légitime d'identifier plusieurs niveaux de discours. Le narrateur fait effectivement mention d'une première série de souvenirs au cours desquels il se remémorait une autre série d'événements qui constituent la métalepse identifiée par Genette. Cependant, ce type de phénomène pose davantage de problèmes d'énonciation que de narratologie. Et, à l'arrivée, la complexité de la notion de métalepse tend à retourner la théorie sur ellemême et provoque une complexification de la taxinomie menant Genette dans la direction d'une synthèse excessive : "toute la Recherche est en fait une vaste analepse pseudo-diégétique au titre des souvenirs du "sujet intermédiaire", aussitôt revendiqués et assumés comme récit par le narrateur final» (DR: 250). Dans ce développement, le 
Genette critique vient tromper le Genette théoricien, qui se laisse en quelque sorte piéger par la complexité du dispositif narratif proustien, à l'instar de Henri Martin-Chauffier, dans un article pour la revue Confluences, qui avait été tenté d'identifier derrière le «je » proustien quatre personnages distincts ${ }^{37}$, modèle dont Genette cherche pourtant à se distancer (DR : 236). Là où la théorie devrait chercher à éclaircir la complexité de la construction du narrateur proustien, le Genette critique semble faire le jeu de cette difficulté d'identification et apporter avec ses outils d'analyse un éloge indirecte au brouillage d'identité en jeu dans le roman. Dans le cas des récits pseudo-diégétiques de seconde main, on retrouve cette démarche que nous pourrions qualifier d' hypercomplexification des phénomènes. Ainsi, considérant l'exemple sans doute le plus marqué de ce type de complexité au sein de la Recherche, Genette commente la prise en charge narrative d'Un Amour de Swann:

En son principe, [Un amour de Swann] est doublement métadiégétique, puisque tout d'abord les détails en ont été rapportés à Marcel par un narrateur et à un moment indéterminés, et ensuite parce que Marcel se remémore ces détails au cours de certaines nuits d'insomnie : souvenirs de récits antérieurs, donc, à partir de quoi, une fois de plus, le narrateur extradiégétique ramasse toute la mise et raconte en son propre nom toute cette histoire survenue avant sa naissance, non sans y introduire de subtiles marques de son existence ultérieure, qui y sont comme une signature et empêchent le lecteur de l'oublier trop longtemps: bel exemple d'égocentrisme narratif. (DR : 250)

Premièrement, postuler que ce récit serait doublement métadiégétique confère une complexité narratologique au texte par l'empilage de deux niveaux de prise en charge, dont le second est, à l'instar des récits intérieurs, quelque peu superflu. Car, certes, le récit a été rapporté au narrateur par une seconde instance tierce, mais, encore une fois, l'activité mnémonique du narrateur proustien qui se « remémore ces détails au cours de certaines nuits d'insomnie " ne constitue pas à proprement parler une «narration». Ils sont effectivement des moments de souvenirs d'un récit, mais ne sont en aucun cas une mise en scène, suffisamment manifeste pour être considérée comme telle, des récits nocturnes que le narrateur se fait à lui-même. Postuler cela mène, secondement, à une interprétation du dispositif visant à mettre en valeur le rôle du narrateur au sein d'un récit dont les modalités tendent en réalité à mettre son énonciation à distance. Il est en effet remarquable que des indices permettent de lui attribuer ce récit, mais il n'empêche que vouloir voir ici un dédoublement du niveau métadiégétique relève de la surinterprétation, et cela relève par conséquent de ce que nous interprétons comme une volonté de légitimation du déploiement théorique par une célébration de l'hypercomplexité de l'objet étudié. Dans les faits, nul indice suffisamment redondant qui pourrait être perçu clairement par le lecteur ne permet d'identifier une quelconque voix originelle derrière la prise en charge énonciative explicite. Il ne fait aucun doute que le récit d'Un Amour de Swann est métadiégétique, mais c'est aller trop loin que de vouloir y reconnaître un enchâssement narratif supplémentaire. Par ailleurs, complexifier ainsi l'objet d'un point de vue théorique tend à rendre la conceptualisation trop spécifique et diminue sa valeur généralisable. Cependant, pour la perspective critique, cette opération d'hypercomplexification parait légitime, car elle sert à singulariser un objet littéraire qui réclame, de toute évidence, la constitution d'un appareillage théorique démesuré. C'est bien là que le double objectif de DR devient problématique. Refusant de choisir entre deux projets qu'il aurait pu mener distinctement, l'option de Genette de servir deux visées conjointes met le projet théorique en péril, tout en alourdissant l'analyse critique. 

sur les relations diégétiques. En effet, le développement visant à établir les catégories d'homo-, hétéro- et autodiégétique se construit dans un aller-retour entre catégorisation théorique et évaluation "génétique " des effets du passage d'une narration à plusieurs niveaux (dans Jean Santeuil) vers le récit «à la première personne » de la Recherche. En termes d'imbrication entre discours critique et propositions théoriques, le sous-chapitre consacré aux problèmes de la «personne» (DR: 251-259) se fait particulièrement problématique. Ici comme ailleurs, le point de départ de Genette est influencé par l'objectif d'analyser la Recherche. En effet, il affirme d'emblée :

Le choix du romancier n'est pas entre deux formes grammaticales, mais entre deux attitudes narratives (dont les formes grammaticales ne sont qu'une conséquence mécanique): faire raconter l'histoire par l'un de ses «personnages», ou par un narrateur étranger à cette histoire. [...]

En tant que le narrateur peut à tout instant intervenir comme tel dans le récit, toute narration est, par définition, virtuellement faite à la première personne. (DR : 252)

Le déplacement du problème linguistique de l'énonciation narrative vers celui de l'« attitude auctoriale » prend très nettement sa source dans les observations génétiques qui mobilisent quasiment l'ensemble du chapitre. Genette propose ici une posture théorique fonctionnant en partie sur une inférence concernant l'existence qu'il existerait plusieurs variantes possibles du récit parmi lesquels l'auteur aurait à choisir, et non sur ce que le texte propose au lecteur. Or, seuls quelques rares exemples littéraires - parmi lesquels la Recherche, qui comprends deux «versions " d'un même récit, ou La Peste de Camus -, permettent de partir d'un tel postulat. Envisager, comme Genette, que «toute narration est, par définition, virtuellement faite à la première personne ", implique de se contraindre à construire une voix narrative même quand celle-ci ne se manifeste pas spécifiquement par le biais d'un embrayage explicite des énoncés fictionnels. Cependant, en prenant en compte le fait que ce principe découle d'une réflexion sur l'œuvre de Proust, le raccourci pris dans cette proposition est, bien que théoriquement fragile, envisageable du point de vue du discours critique. En effet, le cheminement menant à cette remarque tire son origine de l'analyse du « triomphe du pseudo-diégétique » quelques pages plus tôt: "Proust avait goûté dans Jean Santeuil aux plaisirs désuets du métadiégétique, tout se passe comme s'il avait juré de n'y plus revenir, et de se réserver (ou de réserver à son porte-parole) la totalité du discours narratif » (DR: 250). Outre l'interprétation biographique quelque peu audacieuse, on voit que Genette fonde son approche sur ce qu'il analyse comme un bouleversement formel entre la rédaction de Jean Santeuil et la création de la Recherche. Ici, la critique proustienne influence le développement théorique en le conduisant à tabler sur des présupposés issus de sa propre interprétation génétique d'un corpus spécifique. Le passage d'une narration présentant un feuilletage de plusieurs narrateurs enchâssés dans Jean Santeuil au récit à la " première personne» de la Recherche influence la perspective du critique, qui envisage la construction du narrateur proustien comme l'achèvement d'un projet romanesque engagé à l'occasion d'un texte antérieur. L'accent placé sur la figure d'un narrateur, qui serait toujours présent derrière n'importe quel récit, découle probablement en grande partie de la visée d'une analyse fondée sur l'œuvre de Proust, et qui ne prend que peu acte de la diversité effective des formes narratives en général. En outre, cette partie du développement, révèle la tension manifeste entre la notion de narrateur et d'auteur. On l'a vu, la démarche de Genette tient compte de l'œuvre antérieure de Proust pour 
expliquer des phénomènes de narration présents dans la Recherche. Or, il serait légitime de se demander si, comme il le présuppose dans l'introduction, "l'acte de Marcel racontant sa vie passée [ne se confond pas, au moins un peu] avec l'acte de Proust écrivant la Recherche du temps perdu.» (DR : 73) Aussi, comme le relève à juste titre Sylvie Patron:

Sous le terme de narrateur, Genette rapproche donc deux instances que je n'hésiterai pas à qualifier d'incompatibles: une instance effective de production d'un récit (c'est l'auteur dans son acception poétique, désormais simplement l'auteur) et la représentation fictive ou fictionnelle qui peut être donnée de cette instance dans le récit lui-même (c'est, par exemple, le narrateur d'À la Recherche du temps perdu $u^{38}$.

On voit poindre ici l'un des nœuds du problème, que Genette révèle malgré lui. À vouloir mélanger critique proustienne et narratologie générale, il se laisse prendre au piège de l'explication de l'œuvre que disqualifie son approche théorique. Le refus de vouloir entendre la voix d'un quelconque auteur dans sa construction de la narration est en fait court-circuitée par son étude de Proust, qui réintroduit, au sein de la rigueur narratologique, la subjectivité des interprétations critiques. Aussi, du point de vue théorique avec lequel nous approchons ce texte, le problème de la notion de narration paie le prix de l'indécision originelle de Genette entre critique et théorie.

Le problème fondamental de DR se pose dès lors que l'on désire l'envisager comme un projet purement théorique. L'objectif critique envisagé par Genette pose problème parce qu'il l'amène à se focaliser sur un objet spécifique qui le contraint à s'attarder sur des complexités propres, si ce n'est à son objet, tout du moins à une certaine catégorie d'objets. Aussi, Mieke Bal faisait-elle remarquer cela au sujet spécifique de la narration :

Le type de récit le plus répandu, le récit où le narrateur est absent ou invisible (le récit raconté «à la troisième personne»), n'a pas de place dans la typologie. Qui sait, par exemple, si le narrateur de La chatte, quand il décrit au début du roman une soirée paisible en famille, est déjà au courant de la fin malheureuse du roman? La question me semble sans pertinence aucune, car ce narrateur, étant absent, se soustrait à l'intérêt du roman. Cette restriction du champ d'application de la typologie ne la dévalorise pas en soi - pour les récits à narrateur visible, elle est d'une grande valeur analytique - mais il est regrettable qu'elle ne couvre pas l'objet entier de la narratologie, qu'elle ne relève donc pas d'une théorie générale du récit ${ }^{39}$.

Comme le remarque Bal, la focalisation de Genette sur l'œuvre de Proust tend à évacuer trop de paramètres pour constituer les fondements d'une narratologie générale. Dans le but de décrire la narration proustienne, Genette est obligé de construire un système sur des bases qui ne peuvent refléter tous les enjeux de la narration, ne serait-ce que du point de vue de la littérature ${ }^{40}$. Qu'il s'agisse du temps, du niveau ou de la relation, tout le chapitre dédié à la voix montre que l'objectif critique complique sensiblement la construction théorique et tend à produire une théorie de la narration qu'il serait nécessaire de rediscuter et resituer au croisement des différentes problématiques qui participent à sa genèse.

\section{Conclusion}

Les critiques adressées par l'école non-communicationnaliste de la narratologie ont, à juste titre, pointé du doigt cette obsession du narrateur, que nous avons mise en relation 
avec la perspective critique sur l'œuvre de Proust dans DR. Il semble toutefois que peu de ces critiques se soient interrogés sur les raisons qui sont à l'origine de la centralité de cette notion dans l'échafaudage théorique de Genette. Ce que nous avons montré ne visait à disqualifier aucun des discours appartenant à l'un et l'autre de ces deux paradigmes. Au contraire, par le biais de notre étude détaillée du texte de Genette, nous pensons avoir montré que les enjeux sont multiples et que, tant les attaques qu'il a pu connaître que l'enthousiasme quelque peu aveugle qu'il a pu provoquer dans sa récupération postérieure, mériteraient d'être réinterrogés à l'aune d'une relecture plus précise au texte, qui est à appréhender comme un projet critique à part entière, et non simplement comme un réservoir de notions théoriques.

Comme nous en avons fait l'esquisse au cours de la première partie de notre développement, la narratologie genettienne demanderait une réflexion épistémologique plus aboutie ${ }^{41}$. La méthode à laquelle il s'astreint est non seulement à prendre en compte, mais aussi à recontextualiser à la lumière des différents paramètres constitutifs du moment intellectuel des années 1960-70, qui influent très manifestement sur son texte. Il est certes fortement influencé par le paradigme structuraliste, mais il se positionne également à l'encontre de certains de ses principes en proposant une approche quasi expérimentale, qui rapproche geste théorique et lecture critique.

Aussi, prendre en considération le double projet de Genette permet de saisir un grand nombre de ses présupposés et d'en comprendre le développement. La part critique de son projet influence directement son discours théorique et nécessite donc d'être pris en compte pour mieux comprendre les enjeux qui sous-tendent la genèse de ses concepts. Comme nous l'avons souligné, dans DR aucun des deux projets, critique ou théorique, n'est privilégié, et il nous semble donc qu'un commentaire de l'outillage genettien ne devrait pas ignorer ce paramètre en quelque sorte méthodologique, plus qu'épistémologique.

41 Car, comme nous l'avons montré au cours de notre étude sur la construction des outils descriptifs de la voix, il est manifeste que, pour les plus complexes d'entre eux, l'influence proustienne est décisive. En effet, si notre analyse a pu mettre en cause, dans certains cas, la rentabilité d'une catégorie théorique trop étroitement liée à l'œuvre de Proust, cela n'implique pas pour autant sa disqualification (nous n'en avons ni la prétention ni, évidemment, l'autorité). En revanche, ce que nous avons tenté de montrer, c'est que ces catégories nécessitent de prendre en compte l'influence de Proust sur la genèse théorique des paramètres du récit, notamment pour ceux qui concernent la narration. Aussi, le présent travail laisse-t-il de côté plusieurs autres réflexions possibles, auxquelles il serait pertinent de prêter attention. Poursuivant une perspective théorique, notre point de vue nous a, en effet, poussé du côté de l'évaluation de cette part de l'essai genettien. Pourtant, il semble que peu de travaux ont été produits quant à l'objectif critique affiché par DR. Ainsi, se pencher sur cet aspect du texte pourrait sans doute produire des résultats intéressants et participerait à remettre en lumière cette part très peu envisagée de l'essai de Genette. Par ailleurs, nous n'avons eu que trop peu l'occasion de développer notre propos quant à l'inscription, pourtant très singulière, de Genette au sein du contexte intellectuel des années 1960-70. Il nous semble pourtant qu'une étude des conditions d'émergence d'un tel texte en 1972 participerait à éclairer différemment cet objet et apporterait une contribution solide à l'épistémologie de la narratologie. Car, que l'on s'oppose à ce texte ou que l'on en fasse l'éloge, son étude détaillée s'avère être aujourd'hui toujours aussi nécessaire, tant dans son contexte que dans sa forme, car il 
concentre, à notre sens, bien plus que d'autres textes de la discipline, peut-être tout ce que le projet narratologique (au sens très français de ce terme) a de complexe et d'angles morts, et qu'il est plus que jamais essentiel de comprendre pour continuer l'entreprise des théories du récit.

\section{BIBLIOGRAPHY}

\section{Sources}

GENETTE, Gérard, « Discours du récit (Essai de méthode) » in, Figures III, Paris : Seuil, coll.

« Poétique », 1972, p. 65-274.

- Nouveau discours du récit, Paris : Seuil, coll. « Poétique », 1983.

- Discours du récit, Paris : Seuil, coll. « Point », 2007 [1972 et 1983].

\section{Littérature secondaire}

BACHELLIER, Jean-Louis, « La poétique lézardée. Figures III, de Gérard Genette », in, Littérature, Décembre 1973, p. 107-113.

BAL, Mieke, Narratologie. Essai sur la signification narrative de quatre romans modernes, Paris : Klinksieck, 1977.

BANFIELD, Ann, Phrases sans parole. Théorie du récit et du style indirect libre, Paris : Seuil, coll. «Philosophie générale », 1997 [orig. Angl. 1982].

BARTHES, Roland, Critique et vérité, Paris : Seuil, coll. « Point », 1966.

- «Introduction à l'analyse structurale du récit », in, Communications, nº 8, 1966, p.1-24.

- « La Mort de l'auteur », in, Le Bruissement de la langue, Paris : Seuil, coll. « Point », 1984 [1968], p. 64-69.

COMPAGNON, Antoine, Le Démon de la théorie, Paris : Le Seuil, coll. « Point », 1998.

DOSSE, François, Histoire du structuralisme, 2 t., Paris : La Découverte, coll. « Textes à l'appui », 1991-1992.

DOUBROWSKY, Serge, Pourquoi la nouvelle critique ? Critique et subjectivité, Paris : Mercure de France, 1966.

GENETTE, Gérard, « Critique et poétique » in, Figures III, Seuil, coll. « Poétique », 1972, p. 9-11.

- « Frontières du récit », in, Communications, n8, 1966, p.152-163.

- « Poétique et Histoire ». in, Figures III, Seuil, coll. « Poétique », 1972, p.13-20.

JONES, Robert Emmet, Panorama de la nouvelle critique en France : de Gaston Bachelard à JeanPaul Weber, Paris : Sedes, 1968.

MAINGUENEAU, Dominique, Contre Saint Proust, ou la fin de la littérature, Paris : Belin, 2006.

- Le Discours littéraire. Paratopie et scène d'énonciation, Paris : Armand Colin, coll. « U Lettres », 2004. 
MARTIN-CHAUFFIER, Louis, " Proust et le double "Je" de quatre personnes », in Confluences, Problèmes du roman, $n^{\circ}$ 21, Aout 1943, p. 55-69.

PATRON, Sylvie, Le Narrateur. Introduction à la théorie narrative, Armand Colin, coll. « U. Lettres », 2009.

- «Sur l'épistémologie de la théorie narrative (narratologie et autres théories du récit de fiction) », Les Temps Modernes, n635, novembre-décembre 2005 - janvier 2006, p.262-285

PHILIPPE, Gilles, « Présentation », in, Langue française. L'ancrage énonciatif des récits de fiction, $\mathrm{n}^{\circ} 128,2000$, p. 3-8.

PROUST, Marcel, « Lettre du 23 février 1913 », in, Correspondances de Marcel Proust. Tome XIII : 1913, sous la dir. de Philip Kolb, Paris : Plon, 1984, p. 91-92.

RABATEL, Alain, Homo Narrans. Pour une analyse énonciative et interactionnelle du récit. Les points de vue et la logique de la narration, t. I, Limoges : Lambert-Lucas, 2008.

- Homo Narrans. Pour une analyse énonciative et interactionnelle du récit. Dialogisme et polyphonie dans le récit, t. II, Limoges : Lambert-Lucas, 2008.

TODOROV, Tzvetan, Grammaire du Décameron, Paris : Mouton, 1969.

- « Les catégories du récit littéraire », in, Communications, n8, 1966, p.125-151.

- Qu'est-ce que le structuralisme? Poétique, t. II, Paris: Seuil, coll. « Point », 1968. WAGNER, Frank, « Aimez-vous "Genette” ? (Éloge de la poétique cum grano salis) »in, Fabula-LhT, n¹0, Décembre 2012, en ligne (consulté le 21.08.2015): url: http://www.fabula.org/lht/10/ wagner.html.

\section{NOTES}

1. Marcel Proust, «Lettre du 23 février 1913 », in, Correspondances de Marcel Proust. Tome XIII : 1913, sous la dir. de Philip Kolb, Paris : Plon, 1984, p. 91-92.

2. Gérard Genette, Nouveau discours du récit, Paris : Seuil, coll. « Poétique », 1983.

3. Gérard Genette, «Discours du récit (Essai de méthode)» in, Figures III, Paris: Seuil, coll. «Poétique », 1972, p. 65-274. Au vu du grand nombre de références faites dans le présent travail au sujet de cet article, nous en ferons désormais mention par l'acronyme DR, suivi de la page concernée, dans le corps du texte, afin de limiter la multiplication des notes.

4. « [L]e propos, conçu si je ne m'abuse pendant l'hiver (février-avril) 1969 à New Harbour, Rhode Hampshire, où je me trouvais fréquemment retenu "chez moi" par les congères, d'éprouver et systématiser quelques catégories, déjà entrevues çà et là, sur le seul texte dont je disposais "à domicile" : les trois volumes Pléiade de la Recherche, et sur les débris erratiques d'une mémoire littéraire déjà passablement sinistrée. » (Gérard Genette, Nouveau discours du récit, op. cit., p. 9.)

5. Sylvie Patron va jusqu'à affirmer que : «Pendant longtemps, la notion de théorie narrative a semblé être coextensive à sa version narratologique; et encore aujourd'hui, on imagine volontiers que le concept de narrateur lui est absolument nécessaire. Cette liaison intrinsèque, consubstantielle, entre la théorie narrative et le narrateur se fonde sur une autorité unique et dans l'ensemble peu discutée : "Discours du récit. Essai de méthode " de Gérard Genette, publié dans Figures III en 1972. " (Sylvie Patron, Le Narrateur. Introduction à la théorie narrative, Armand Colin, coll. «U. Lettres », 2009, p. 30)

6. À l'ouverture du chapitre consacré à la voix dans le Nouveau discours du récit Genette relève : "Le chapitre de la voix est sans doute celui qui a provoqué pour moi les discussions les plus cruciales, au moins à propos de la catégorie de la personne. » (Gérard Genette, op. cit., p. 52) 
7. Pour une introduction au désaccord entre narratologie communicationnelle et non communicationelle, voir Gilles Philippe, «Présentation », in, Langue française. L'ancrage énonciatif des récits de fiction, $\mathrm{n}^{\circ} 128,2000$, p. 3-8.

8. Ann Banfield, Phrases sans parole. Théorie du récit et du style indirect libre, Paris: Seuil, coll. «Philosophie générale », 1997 [orig. angl. 1982].

9. Sylvie Patron, op. cit.

10. «Ce livre est né d'une indignation. J'ai été indignée en relisant, bien des années après une première lecture qui était une lecteur scolaire, les deux pages que Gérard Genette consacre à l'ouvrage d'Ann Banfield, Unspeakable Senteces: Narration and Representation in the Language of Fiction (1982), dans son ouvrage, Nouveau discours du récit (1983). [...] Prendre au sérieux Genette comme théoricien (exactement comme métathéoricien) aujourd'hui n'est pourtant pas très facile.» (ibid., p. 9)

11. Dominique Maingueneau rappelle à cet égard que : «Cette évolution s'est accompagnée d'une transformation dans les modes de légitimation de ceux qui traitaient de littérature. Ne pouvant se reconnaître ni dans la figure du commentateur éminemment subjectif (dans les années 1960 on se plaisait à le dire "impressionniste"), ni dans celle de l'érudit méticuleux, le "nouveau critique " prétendait cumuler la légitimité universitaire du modélisateur et de l'épistémologue avec le charisme de l'essayiste, les pouvoirs de l'homme de science et ceux de l'homme d'écriture. » (Dominique Maingueneau, Contre Saint Proust, ou la fin de la littérature, Paris: Belin, 2006, p.112)

12. Roland Barthes, Critique et vérité, Paris : Seuil, coll. « Point », 1966, p.61-82.

13. Cette démarche a constitué le moteur d'action principal de la nouvelle critique, comme le relève Maingueneau: «Ce qu'on a appelé "nouvelle critique" est une alliance entre des approches divergentes à bien des égards, mais qui avaient toutes un ennemi commun, l'histoire littéraire, qui s'est tenue à distance des sciences humaines, en dépit de quelques vagues tentatives de rapprochement, en particulier chez G. Lanson, intéressé par la sociologie naissante. L'histoire littéraire constituait un repoussoir si puissant que l'union des tenants de la nouvelle critique se réalisait sans difficulté : il suffisait de promouvoir une approche "interne " des œuvres et de récuser les démarches atomisantes pour passer pour "structuraliste".» (Dominique Maingueneau, Le Discours littéraire. Paratopie et scène d'énonciation, Paris : Armand Colin, coll. « U Lettres ", 2004, p. 18)

14. Roland Barthes, op.cit., p.61-62.

15. Tzvetan Todorov, Qu'est-ce que le structuralisme? t.2, Poétique, Paris : Seuil, coll. « Point », 1968, p.19.

16. Gérard Genette, "Critique et poétique », in, Figures III, Paris : Seuil, coll. « Poétique », 1972, p.9-11 et « Poétique et Histoire », in, Figures III, Paris : Seuil, coll. « Poétique », 1972, p. 13-20.

17. Voir à cet égard, l'article fondateur proposé par Barthes dans le huitième numéro de la revue Communications exposant les enjeux principaux d'une discipline à fonder. (Roland Barthes, "Introduction à l'analyse structurale du récit», in, Communications, n 8, 1966, p. 1-24) Par ailleurs le terme de «science » est explicitement employé par Tzvetan Todorov lorsqu'il propose de déterminer comme «narratologie » cette «science nouvelle » qu'était jusque-là, l'analyse structurale du récit. Tzvetan Todorov, Grammaire du Décameron, Paris : Mouton, 1969, p. 10

18. La première phrase est on ne peut plus formelle sur ce point : «L'objet spécifique de cette étude est le récit dans À la recherche du temps perdu. » (DR : 67)

19. Dominique Maingueneau, op. cit.

20. Ibid., p.35

21. «La Recherche, moins qu'aucune autre, ne peut être considérée comme une cuvre close, et il est donc toujours légitime et parfois nécessaire d'en appeler pour comparaison du texte "définitif" à telle ou telle de ses variantes. (DR: 67) 
22. Ainsi que l'explique Maingueneau : "Témoin de cette ambiguïté, le succès que connaît alors le Contre Sainte-Beuve de Proust. Si cet ouvrage, qui s'inscrit pourtant dans la continuité de l'idéalisme du début du XIX ${ }^{\mathrm{e}}$ siècle, est perçu comme novateur, c'est précisément parce qu'il porte l'attaque au cœur de l'appareil de l'histoire littéraire (“l'homme”, “œœuvre” et "le milieu") et qu'il annonce la "critique thématique", au firmament dans les années 1960 et qui tranche sur la stylistique grammaticale, alors dominante dans l'appareil scolaire française. " (Dominique Maingueneau, Le Discours littéraire. Paratopie et scène d'énonciation, op. cit. p. 20)

23. Voir, Roland Barthes, « La Mort de l'auteur », in, Le Bruissement de la langue, Paris : Seuil, coll. «Point », 1984 [1968], p. 64-69.

24. Ann Banfield en fait l'interprétation suivante: «Le besoin de se rapporter à un narrateur même en de toute première personne s'explique par le manque de cadre théorique permettant de penser le texte soit comme un tout unifié, soit comme un objet intentionnel. Remplacer le terme, devenu tabou, d'“auteur" par celui de "narrateur" pour représenter l'unité hypothétique, ce n'est jamais qu'une astuce terminologique pour essayer de biaiser les limites étroites qu'impose à la théorie du récit la notion essentiellement empiriste du texte héritée de la Nouvelle Critique.» (Ann Banfield, op. cit., p.279)

25. «Ce que j'appelle la fréquence narrative [...] a été jusqu'ici fort peu étudié par les critiques et les théoriciens du roman. » (DR : 145)

26. Mieke Bal, Narratologie. Essai sur la signification narrative de quatre romans modernes, Paris: Klinksieck, 1977, p. 6.

27. Telles que développées dans Tzvetan Todorov, "Les catégories du récit littéraire ", Communications, $\mathrm{n}^{\circ} 8,1966, \mathrm{p} .125-151$.

28. «L'instance narrative » (DR: 225-227).

29. « Temps de la narration» (DR: 228-238).

30. « Niveaux narratifs » (DR: 238-251\}); « Le récit métadiégétique »(DR: 241-243) ; « Métalepses » (DR: 243-246) ; «De Jean Santeuil à la Recherche ou le triomphe du pseudo-diégétique » (DR: 246-251)

31. « Personne » (DR: 251-261) ; « Héros / Narrateur » (DR: 259-261).

32. « Fonctions du narrateur » (DR: 261-265).

33. « Le narrataire» (DR: 265-267)

34. Le premier ne constitue qu'un établissement typologique se détachant du développement général, de même que le narrataire constitue davantage une ouverture qu'un étape de l'argumentation.

35. Gérard Genette, Nouveau discours du récit, op. cit., p. 55-64

36. Les deux exemples qu'il fournit dans un premier temps sont soit imaginaire : «comme si le marquis de Renoncourt, après avoir reconnu qu'il tient de des Grieux lui-même l'histoire de ses amours [...] reprenait ensuite la parole pour raconter cette histoire lui-même » (DR : 245), nous soulignons) ; soit, tiré du Théétète de Platon, texte dont l'économie «narrative » est, à bien des égards, difficilement comparable avec un corpus de littérature narrative fictionnelle.

37. Louis Martin-Chauffier, "Proust et le double "Je" de quatre personnes", in, Confluences, Problèmes du roman, ${ }^{\circ} 21$, Août 1943, p.55-69.

38. Sylvie Patron, op. cit., p.280.

39. Mieke Bal, op. cit., p.30.

40. Il est évident que la focalisation sur la problématique du narrateur pose également d'énormes problèmes pour un élargissement du champ de la narratologie aux narrations visuelles ou audiovisuelles.

41. Quelques bases générales d'une épistémologie de la narratologie sont posées par Sylvie Patron dans « Sur l'épistémologie de la théorie narrative (narratologie et autres théories du récit de fiction) », Les Temps Modernes, n635, novembre-décembre 2005 - janvier 2006, p.262-285. 


\section{ABSTRACTS}

The author defends the hypothesis that the generation of the narrative voice's concept in the " Discours du récit » by Gérard Genette would be largely conditioned by the complex maintained relationship with its object of study: À la recherche du temps perdu from Marcel Proust. Assuming that Genette's article is built around a perspective in tension between theory and literary criticism, reflection is the influence established by the choice and treatment of this corpus on the construction of theoretical tools. Genette's methodology is therefore well studied in the intellectual context of the late sixties, focusing on objectives explicitly stated in his article. Furthermore, the author proposes an analysis of the application of this method in the "Discours du récit " by showing that both in the general construction of the article, and in the section specifically devoted to the "Voix ", critical target of the Proustian narrative complicates the theoretical establishment and directs the genettian narratology to a communicative representation of the narrative.

L'auteur défend l'hypothèse selon laquelle la génération de la notion de voix narrative dans «Discours du récit» de Gérard Genette serait en grande partie conditionnée par le rapport complexe entretenu avec son objet d'étude: À la recherche du temps perdu de Marcel Proust. Partant du principe que l'article de Genette se construit autour d'une perspective en tension entre théorie et critique littéraire, la réflexion vise l'influence exercée par le choix et le traitement de ce corpus sur la construction de l'outillage théorique. La méthodologie de Genette est ainsi étudiée au sein du contexte intellectuel de la fin des années soixante, en mettant l'accent sur les objectifs explicitement exposés dans son article. En outre, l'auteur propose l'analyse de l'application de cette méthode dans le « Discours du récit » en démontrant que, tant dans la construction générale de l'article, que dans la partie spécifiquement consacrée à la «Voix », la visée critique du récit proustien complexifie l'établissement théorique et dirige la narratologie genettienne vers une représentation communicationnelle du récit.

\section{INDEX}

Geographical index: France

Mots-clés: narrateur, voix narrative, Genette Gérard, nouvelle critique, Proust Marcel, narratologie non communicationnelle

Chronological index: XXe siècle

\section{AUTHOR}

\section{OLIVIER STUCKY}

Université de Lausanne

Olivier Stucky est étudiant en master de Lettres à l'Université de Lausanne en sections de 
Français moderne et d'Histoire et esthétique du cinéma. Il rédige actuellement, sous la direction du prof. Alain Boillat, un mémoire de maîtrise sur l'epistémologie de la narratologie filmique, se concentrant sur le problème de la construction théorique de la notion de narrateur dans cette discipline. 